\title{
On Quotient Structures of Fuzzy Multiset Finite Automata
}

\author{
Anand P. Singh ${ }^{a}$, M. K. Dubey ${ }^{b}$ and I. Perfilieva ${ }^{a}$ \\ ${ }^{a}$ University of Ostrava \\ Institute for Research and Applications of Fuzzy Modeling \\ NSC IT4Innovations, 30. dubna 22, 70103 Ostrava 1, Czech Republic, \\ anand.singh@osu.cz,irina.perfilieva@osu.cz \\ ${ }^{b}$ Department of Mathematics and Statistics, \\ Manipal University Jaipur, Jaipur-303007, Rajasthan, India, \\ maheshdubey6@gmail.com
}

\begin{abstract}
The objective of this paper is to characterize the quotient structures of fuzzy multiset finite automata. We introduce different congruence relation on multiset associated with fuzzy multiset finite automata and show that each congruence relation associates a semigroup with the fuzzy multiset finite automata. We also discuss the charaterization of fuzzy multiset finite automata by defining an admissible relation.
\end{abstract}

Keywords: Multiset; fuzzy multiset finite automaton; congruence relation; admissible relation; homomorphism.

\section{Introduction}

The finite automaton is one of the well-known mathematical model of computation in the computing science. After the introduction of fuzzy set by Zadeh [25], the notion of classical automaton has been generalized to the fuzzy case in the paper of Wee [23] and Santos [14]. A number of research presented in (cf., [7, 9, 10, 13, 24]) highlights the development on fuzzy automata.

A multiset is a generalization of the concept of a set, which allows multiple instances for each of its elements [2]. In the recent years, the diverse applications of multiset has been shown in various areas such as mathematics, computer science, biology and biochemistry (cf., $[1,3,11]$ ). It has been shown that the concept of multiset is useful in the characterization of automata theory and then the concepts of multiset grammars and multiset finite automata are introduced and studied ( $[3,4])$. In another direction, author of (cf., [4]) studied the theory of Mealy multiset automata in membrane computing. Further, a category theoretic approach has been used to discuss the algebraic and coalgebraic properties of Mealy type multiset automata [5].

In recent years the concept of finite automata has been generalized to fuzzy finite automata having memebership values in more general structures such as distributive lattice, complete residuated lattice and lattice ordered monoids. Motivated by this generalization Wang, Yin and $\mathrm{Gu}$ [21] introduced the notion of fuzzy multiset finite automata as a generalization of multiset finite automata. In [21], it is shown that a fuzzy multiset finite automata accepts the fuzzy multiset regular grammar, which is generated from a fuzzy multiset language. After that Tiwari et al. [19], studied the determinization (i.e., behavior of equaivalence of fuzzy multiset finite automaton and deterministic fuzzy multiset finite automata) and minimal realization problems of fuzzy multiset finite automata. Sharma et al. (c.f., [16-18]) have discussed the algebraic study of fuzzy multiset finite automata and fuzzy multiset languages. The minimization of fuzzy multiset finite automata with membership value in a distributive lattice were studied by Wang et al. [22] and a simplified version of fuzzy multiset automata has been discussed in [20]. Recently, Pal et al. [12] applied the Brzozowski's algorithm to study the minimal realization of an $L$-valued multiset language. However, the study of quotient structure based fuzzy multiset finite automata is still missing. In order to give deeper insight into the algebraic study of fuzzy multiset finite automata, in this paper we discuss the quotient structures of fuzzy multiset finite automata. For this purpose, we define a congruence relation on multiset in such a way that each associate a semigroup with a fuzzy multiset finite automata. We also introduce an admissible relation on a given fuzzy multiset automata and give its characterization. Interestingly, we establish an isomorphism between a fuzzy multiset finite automata and the quotient structure on another fuzzy multiset finite automata. 
This paper is organized as following. Section 2, is introductory and contains the basic notions of multiset, multiset finite automata and fuzzy multiset finite automata. Section 3 contains the main result of this paper. We introduce a congruence relation on fuzzy multiset finite automata and show that each congruence relation associates a semigroup with the fuzzy multiset finite automata. Furthermore, we define the admissible relation on a fuzzy multiset finite automata and discuss its characterization.

\section{Preliminaries}

In this section we review the concepts of multisets, multiset finite automata, and fuzzy multiset finite automata. Below we recall the definition of multiset and multiset finite automata from $[2,6]$.

Definition 2.1 Let $\Sigma$ be a finite set of alphabet and $N$ be the set of non-negative integers. Then a map $u: \Sigma \rightarrow$ $N$ is called a multiset over alphabet $\Sigma$.

Given a finite set of alphabet $\Sigma$, the norm of $u$ is defined by $|u|=\sum_{a \in \Sigma} u(a)$. We shall denote by $\Sigma^{\oplus}$, the set of all multisets over $\Sigma$. The multiset $0_{\Sigma} \in \Sigma^{\oplus}$ is defined by $0_{\Sigma}(a)=0, \forall a \in \Sigma$. Further, a multiset $b \in \Sigma^{\oplus}$, denoted by $\langle b\rangle$ is called singleton multiset, if for each $a \in \Sigma$, it has the following form

$$
<b>(a)= \begin{cases}1 & \text { if } b=a \\ 0 & \text { otherwise }\end{cases}
$$

Let $\Sigma^{\oplus}$ be the set of all multisets over $\Sigma$. Then for multisets $u, v \in \Sigma^{\oplus}$, the operations of inclusion $\subseteq$, addition $\oplus$, and difference $\ominus$ on multiset $\Sigma^{\oplus}$ are defined as follows.

$$
\begin{array}{r}
u \subseteq v \text { if } u(a) \leq v(a),(u \oplus v)(a)=u(a)+v(a), \\
(u \ominus v)(a)=\vee(0, u(a)-v(a)), \forall a \in \Sigma .
\end{array}
$$

Furthermore, $u \subset v$ if $u \subseteq v$ and $u \neq v$. Also, for $A, B \subseteq \Sigma^{\oplus}, A \oplus B=\cup\{u \oplus v \mid u \in A, v \in B\}$. Obviously, $\Sigma^{\oplus}$ is a commutative monoid with identity element $0_{\Sigma}$ with respect to the binary operation $\oplus$.

For a given alphabet $\Sigma$, let $\bar{\Sigma}=\{\langle a\rangle: a \in \Sigma\}$. Consider $\Sigma=\{a, b, c\}$, the multiset $u=(1 / a, 2 / b, 0 / c)$ can be expressed as $\langle a\rangle \oplus\langle b\rangle \oplus\langle b\rangle$.

Below, we recall the concept of multiset finite automata from [6]

Definition 2.2 $A$ nondeterministic multiset finite automaton (in short NMA) is a triple $\mathscr{M}=(Q, \Sigma, d)$, where $Q$ and $\Sigma$ are nonempty finite sets called the state-set and input-set, respectively and $d: Q \times \Sigma^{\oplus} \rightarrow$ $2^{Q}$ is a map called state transition map.

A configuration of a nondeterministic multiset finite automaton $\mathscr{M}$ is a pair $(p, u)$, where $p$ and $u$ denote current state and current multiset, respectively. The transition in a multiset finite automaton are described with the help of configurations. The transition from configuration $(p, u)$ leads to configuration $(q, v)$ if there exists a multiset $w \in \Sigma^{\oplus}$ with $w \subseteq u, q \in d(p, w)$ and $v=u \ominus w$, and is denoted by $(p, u) \rightarrow(q, v)$.

Definition 2.3 A nondeterministic multiset finite automaton $\mathscr{M}=(Q, \Sigma, d)$ is said to be deterministic multiset finite automaton (in short DMA) if $|d(p, u)| \leq 1, \forall q \in Q$ and $u \in \Sigma^{\oplus}$.

Definition 2.4 Let $\mathscr{M}=(Q, \Sigma, d)$ and $\mathscr{M}^{\prime}=$ $\left(Q^{\prime}, \Sigma, d^{\prime}\right)$ be two nondeterministic multiset finite automata. A map $h: Q \rightarrow Q^{\prime}$ is said to be homomorphism from $\mathscr{M}$ to $\mathscr{M}^{\prime}$ if $h(d(p, u))=d^{\prime}(h(p), u)$.

Since Zadeh [25], introduced the notion of fuzzy set, further studies have been generalized with values from more general structures. Throughout this paper, we denote $L$ as a distributive lattice with the least element $0_{L}$ and the largest element $1_{L}$. We use $\vee$ to represent the finite supremum operation, and $\wedge$ to represent the finite infimum operation. We shall denote by $L^{X}$ the set of all fuzzy subsets of $X$ (i.e. maps $\lambda: X \rightarrow L$ ). Furthermore, support of fuzzy set $\lambda$ is defined as $\operatorname{supp}(\lambda)=\left\{x \in X: \lambda(x)>0_{L}\right\}$. For a nonempty set $X$, a fuzzy relation $R$ on $X$ is a fuzzy subset of $X \times X$ (i.e. a map $R: X \times X \rightarrow L$ ).

Below, we recall the concept of fuzzy multiset finite from [21] with membership value from $L$.

Definition 2.5 $A$ fuzzy multiset finite automaton (in short, $F M A)$ is a triple $\mathscr{A}=(Q, \Sigma, \delta)$, where $Q$ and $\Sigma$ are nonempty finite sets called the state-set and inputset, respectively $\delta: Q \times \Sigma^{\oplus} \times Q \rightarrow L$ is a map called fuzzy state transition map.

A configuration of fuzzy multiset finite automaton $\mathscr{A}$ is a pair $(p, u)$, where $p$ and $u$ denote current state and current multiset, respectively. The transitions in a fuzzy multiset finite automaton are described with the help of configurations. The transition from configuration $(p, u)$ leads to configuration $(q, v)$ with membership value $l \in L$ if there exists a multiset $w \in \Sigma^{\oplus}$ with $w \subseteq u, \delta(p, w, q)=l$ and $v=u \ominus w$ and is denoted by $(p, u) \stackrel{l}{\rightarrow}(q, v) . \stackrel{l^{\prime}}{\rightarrow} *$ denotes the reflexive and transitive closure of $\stackrel{l^{\prime}}{\rightarrow}$, i.e., for $(p, u),(q, v) \in Q \times \Sigma^{\oplus}$, $(p, u) \stackrel{l^{\prime}}{\rightarrow} *(q, v)$, if for some $n \geq 0$, there exists $(n+1)$ states $q_{0}, \ldots, q_{n}$ and $(n+1)$ multisets 
$u_{0}, \ldots, u_{n}$ such that $q_{0}=p, q_{n}=q, u_{0}=u, u_{n}=v$ and $\left(q_{i}, u_{i}\right) \stackrel{l_{i}}{\rightarrow}\left(q_{i+1}, u_{i+1}\right), \forall i=0, \ldots, n-1$, where $l^{\prime}=\left\{l_{0} \wedge l_{1} \wedge \ldots \wedge l_{n-1}\right\}$. Now, we define $\mu_{\mathscr{A}}\left(\left(p_{1}, u\right) \rightarrow *\left(p_{2}, v\right)\right)=\bigvee\left\{l^{\prime}:(p, u) \rightarrow *\left(p^{\prime}, v\right)\right\}$,

and

$$
\mu_{\mathscr{A}}\left(\left(p_{1}, u\right) \rightarrow *\left(p_{2}, u\right)\right)= \begin{cases}1_{L} & \text { if } p_{1}=p_{2}, \\ 0_{L} & \text { if } p_{1} \neq p_{2} .\end{cases}
$$

Example 2.1 Let $\mathscr{A}=(Q, \Sigma, \delta)$ be fuzzy multiset $f$ nite automata (FMA), where $Q=\left\{q_{1}, q_{2}, q_{3}, q_{4}\right\}, \Sigma=$ $\left\{a_{1}, a_{2}, a_{3}\right\}$ and the fuzzy transition functions are given as;

$$
\begin{aligned}
\delta\left(q_{1},<a_{2}>, q_{3}\right) & =0.3 \\
\delta\left(q_{3},<a_{2}>\oplus<a_{3}>, q_{4}\right) & =0.7 \\
\delta\left(q_{4},<a_{1}>, q_{2}\right) & =0.9 \\
\delta\left(q_{1},<a_{2}>\oplus<a_{3}>, q_{3}\right) & =0.4 \\
\delta\left(q_{3},<a_{2}>, q_{4}\right) & =0.5 .
\end{aligned}
$$

Take $u=<a_{1}>\oplus<a_{2}>\oplus<a_{2}>\oplus<a_{3}>$ and $v=0_{\Sigma}$, then the transition steps $\left(\left(q_{1}, u\right) \rightarrow *\left(q_{2}, v\right)\right)$ are given below;

(i) $\left(q_{1},<a_{1}>\oplus<a_{2}>\oplus<a_{2}>\oplus<a_{3}>\right) \stackrel{0.3}{\longrightarrow}$ $\left(q_{3},<a_{1}>\oplus<a_{2}>\oplus<a_{3}>\right) \stackrel{0.7}{\longrightarrow}\left(q_{4},<a_{1}>\right.$ )$\stackrel{0.9}{\longrightarrow}\left(q_{2}, 0_{\Sigma}\right)$.

(ii) $\left(q_{1},<a_{1}>\oplus<a_{2}>\oplus<a_{2}>\oplus<a_{3}>\right) \stackrel{0.4}{\longrightarrow}$ $\left(q_{3},<a_{1}>\oplus<a_{2}>\right) \stackrel{0.5}{\longrightarrow}\left(q_{4},<a_{1}>\right) \stackrel{0.9}{\longrightarrow}$ $\left(q_{2}, 0_{\Sigma}\right)$.

Thus

$$
\begin{aligned}
\mu_{A}\left(\left(q_{1}, u\right)\right. & \left.\rightarrow *\left(q_{2}, v\right)\right) \\
& =\bigvee\{0.3 \wedge 0.7 \wedge 0.9,0.4 \wedge 0.5 \wedge 0.9\} \\
& =\bigvee\{0.3,0.4\}=0.4
\end{aligned}
$$

The notion of homomorphism between two fuzzy multiset finite automata is given below.

Definition 2.6 Let $\mathscr{A}=(Q, \Sigma, \delta)$ and $\mathscr{A}^{\prime}=\left(Q^{\prime}, \Sigma, \delta^{\prime}\right)$ be two fuzzy multiset finite automata. Then a homomorphism from $\mathscr{A}$ to $\mathscr{A}^{\prime}$ is a map $h: Q \rightarrow Q^{\prime}$ such that for all $q, p \in Q$ and $v \subseteq u \in \Sigma^{\oplus}$ we have,

$\mu_{\mathscr{A}}((q, u) \rightarrow *(p, v)) \leq \mu_{\mathscr{A}^{\prime}}((h(q), u) \rightarrow *(h(p), v))$.

We shall denote by $\mathscr{F} \mathscr{M} \mathscr{A}\left(\Sigma^{\oplus}\right)$, the set of all fuzzy multiset finite automata over a fixed multiset $\Sigma^{\oplus}$.
Definition 2.7 A homomorphism $h$ from $\mathscr{A}$ to $\mathscr{A}^{\prime}$ is said to be strong if for all $q, p \in Q$ and $v \subseteq$ $u \in \Sigma^{\oplus}$ we have, $\mu_{\mathscr{A}^{\prime}}((h(q), u) \rightarrow *(h(p), v))=$ $\bigvee_{r \in Q}\left\{\mu_{\mathscr{A}}((q, u) \rightarrow *(r, v)): h(r)=h(p)\right\}$.

Definition 2.8 A homomorphism (strong homomorphism) $h$ from $\mathscr{A}$ to $\mathscr{A}^{\prime}$ is said to be an isomorphism if $h$ is both one to one and onto.

\section{Main Results}

The construction of quotient structures of fuzzy automata and intuitionistic fuzzy automata have already been studied in $[8,10,15]$. Here, we discuss the quotient structures on fuzzy multiset finite automata. For this purpose, we define a congruence relation on multiset $\Sigma^{\oplus}$ in such a way that each associate a semigroup with a fuzzy multiset finite automata. We also introduce an admissible relation on a given fuzzy multiset automata and give its characterization. Interestingly, we establish an isomorphism between a fuzzy multiset finite automata and the quotient structure on another fuzzy multiset finite automata.

For each $\mathscr{A}=(Q, \Sigma, \delta) \in \mathscr{F} \mathscr{M} \mathscr{A}\left(\Sigma^{\oplus}\right)$. Define a relation $\sim$ on $\Sigma^{\oplus}$ by $u \sim v$ if and only if there exists $w \in \Sigma^{\oplus}$ such that $\mu_{\mathscr{A}}((q, u) \rightarrow *(p, w))=\mu_{\mathscr{A}}((q, v) \rightarrow$ $*(p, w))$, for all $p, q \in Q$. Now we have the following

Proposition 3.1 Let $\mathscr{A}=(Q, \Sigma, \delta) \in \mathscr{F} \mathscr{M} \mathscr{A}\left(\Sigma^{\oplus}\right)$. Then the relation $\sim$ on $\Sigma^{\oplus}$ is a right congruence relation on $\Sigma^{\oplus}$.

Proof. Clearly $\sim$ is an equivalence relation on $\Sigma^{\oplus}$. Now let us assume that $u \sim v$ and $\alpha \in \Sigma^{\oplus}$. Then $\forall q, p \in Q$, we have;

$$
\begin{aligned}
& \mu_{\mathscr{A}}((q, u \oplus \alpha) \rightarrow *(p, w)) \\
&=\bigvee_{r \in Q}\left\{\mu_{\mathscr{A}}((q, u) \rightarrow *(r, \alpha)) \wedge\right. \\
&\left.\mu_{\mathscr{A}}((r, \alpha) \rightarrow *(p, w))\right\} \\
&= \exists r \in Q,\left(\mu_{\mathscr{A}}((q, u) \rightarrow *(r, \alpha)) \wedge\right. \\
&\left.\mu_{\mathscr{A}}((r, \alpha) \rightarrow *(p, w))\right), \\
&= \exists r \in Q,\left(\mu_{\mathscr{A}}((q, v) \rightarrow *(r, \alpha)) \wedge\right. \\
&\left.\mu_{\mathscr{A}}((r, \alpha) \rightarrow *(p, w))\right),(\operatorname{since} u \sim v) \\
&= \bigvee_{r \in Q}\left\{\mu_{\mathscr{A}}((q, v) \rightarrow *(r, \alpha)) \wedge\right. \\
&\left.\mu_{\mathscr{A}}((r, \alpha) \rightarrow *(p, w))\right\} \\
&= \mu_{\mathscr{A}}((q, v \oplus \alpha) \rightarrow *(p, w)),
\end{aligned}
$$

i.e., $\mu_{\mathscr{A}}((q, u \oplus \alpha) \rightarrow *(p, w))=\mu_{\mathscr{A}}((q, v \oplus \alpha) \rightarrow$ $*(p, w))$. Hence $u \oplus \alpha \sim v \oplus \alpha$ or $\sim$ is a right congruence relation on $\Sigma^{\oplus}$. 
Remark 3.1 Note that the above-defined relation $\sim$ on $\Sigma^{\oplus}$, is not a left congruence relation. The proof is easy and we leave it to the reader to verify.

Now for $u \in \Sigma^{\oplus}$, we define a set $[u]=\left\{v \in \Sigma^{\oplus} \mid u \sim v\right\}$ and $S(\mathscr{A})=\left\{[u] \mid u \in \Sigma^{\oplus}\right\}$. We have the following.

Proposition 3.2 Let $\mathscr{A}=(Q, \Sigma, \delta) \in \mathscr{F} \mathscr{M} \mathscr{A}\left(\Sigma^{\oplus}\right)$.. Define a binary operation $\odot$ on $S(\mathscr{A})$ by $[u] \odot[v]=$ $[u \oplus v]$, for all $u, v \in \Sigma^{\oplus}$. Then $\langle S(\mathscr{A}), \odot\rangle$ is a finite semigroup with identity.

Now we define another type of relation $\approx$ on $\Sigma^{\oplus}$ by $u \approx v$ if and only if there exists $w \in \Sigma^{\oplus}$ such that $\mu_{\mathscr{A}}((q, u) \rightarrow *(p, w))>0_{L} \Longleftrightarrow \mu_{\mathscr{A}}((q, v) \rightarrow$ $*(p, w))>0_{L}$, for all $u, v \in \Sigma^{\oplus}$ and $q, p \in Q$.

Proposition 3.3 Let $\mathscr{A}=(Q, \Sigma, \delta) \in \mathscr{F} \mathscr{M} \mathscr{A}\left(\Sigma^{\oplus}\right)$. Then the relation $\approx$ on $\Sigma^{\oplus}$ is a right congruence relation.

Proof. Clearly $\approx$ is an equivalence relation on $\Sigma^{\oplus}$. Now let us assume that $u \approx v$ and $\alpha \in \Sigma^{\oplus}$. Then for all $q, p \in Q$, we have

$$
\begin{aligned}
& \mu_{\mathscr{A}}((q, u \oplus \alpha) \rightarrow *(p, w))>0_{L} \\
& \Longleftrightarrow \quad \bigvee_{r \in Q}\left\{\mu_{\mathscr{A}}((q, u) \rightarrow *(r, \alpha)) \wedge\right. \\
&\left.\mu_{\mathscr{A}}((r, \alpha) \rightarrow *(p, w))\right\}>0_{L} \\
& \Longleftrightarrow \exists r \in Q,\left\{\mu_{\mathscr{A}}((q, u) \rightarrow *(r, \alpha)) \wedge\right. \\
&\left.\mu_{\mathscr{A}}((r, \alpha) \rightarrow *(p, w))\right\}>0_{L} \\
& \Longleftrightarrow \exists r \in Q,\left(\mu_{\mathscr{A}}((q, v) \rightarrow *(r, \alpha)) \wedge\right. \\
&\left.\mu_{\mathscr{A}}((r, \alpha) \rightarrow *(p, w))\right)>0_{L}, \\
&(\operatorname{sinceu} \approx v) \\
& \Longleftrightarrow \bigvee\left\{\mu_{\mathscr{A}}((q, v) \rightarrow *(r, \alpha)) \wedge\right. \\
& r \in Q \\
&\left.\mu_{\mathscr{A}}((r, \alpha) \rightarrow *(p, w))\right\}>0_{L} \\
& \Longleftrightarrow \mu_{\mathscr{A}}((q, v \oplus \alpha) \rightarrow *(p, w))>0_{L} .
\end{aligned}
$$

i.e., $\mu_{\mathscr{A}}((q, u \oplus \alpha) \rightarrow *(p, w))>0_{L} \Leftrightarrow \mu_{\mathscr{A}}((q, v \oplus$ $\alpha) \rightarrow *(p, w))>0_{L}$. Hence $u \oplus \alpha \approx v \oplus \alpha$ or $\approx$ is a right congruence relation on $\Sigma^{\oplus}$.

Remark 3.2 For each $\mathscr{A}=(Q, \Sigma, \delta) \in \mathscr{F} \mathscr{M} \mathscr{A}\left(\Sigma^{\oplus}\right)$ and $u \in \Sigma^{\oplus}$, we define a set $[[u]]:=\left\{v \in \Sigma^{\oplus} \mid u \approx v\right\}$ and $\tilde{S}(\mathscr{A}):=\left\{[[u]] \mid u \in \Sigma^{\oplus}\right\}$.

Proposition 3.4 Let $\mathscr{A}=(Q, \Sigma, \delta) \in \mathscr{F} \mathscr{M} \mathscr{A}\left(\Sigma^{\oplus}\right)$. Define a binary operation $\odot$ on $\tilde{S}(\mathscr{A})$ by

$$
[[u]] \odot[[v]]=[[u \oplus v]], \forall[[u]],[[v]] \in \tilde{S}(\mathscr{A}) .
$$

Then $\langle\tilde{S}(\mathscr{A})$, ○ $\rangle$ is a finite semigroup with identity.

Proof. Clearly, the binary operation (๑) is welldefined and associative. For all $[[u]] \in \tilde{S}(\mathscr{A})$, we have $[[u]] \odot\left[\left[0_{\Sigma}\right]\right]=\left[\left[u \oplus 0_{\Sigma}\right]\right]=[[u]]=\left[\left[0_{\Sigma} \oplus u\right]\right]=$ $\left[\left[0_{\Sigma}\right]\right] \odot[[u]]$. It means that $\left[\left[0_{\Sigma}\right]\right]$ is the identity of $\langle\tilde{S}(\mathscr{A}), \odot\rangle$. The finiteness of $\tilde{S}(\mathscr{A})$ is conclude with the finiteness of $\Sigma$. Hence $\langle\tilde{S}(\mathscr{A}), \odot\rangle$ is a finite semigroup with identity.

From the previous propositions, we have the following.

Proposition 3.5 Let $\mathscr{A}=(Q, \Sigma, \delta) \in \mathscr{F} \mathscr{M} \mathscr{A}\left(\Sigma^{\oplus}\right)$ and $h: S(\mathscr{A}) \rightarrow \tilde{S}(\mathscr{A})$ be map such that $h([u])=[[u]]$, for all $[u] \in S(\mathscr{A})$. Then $h$ is homomorphism of $S(\mathscr{A})$ onto $\tilde{S}(\mathscr{A})$.

Proof. Let $u, v \in \Sigma^{\oplus}$ and $[u]=[v]$. Then for all $p, q \in Q$ and there exsts $w \in \Sigma^{\oplus}$ such that $\mu_{\mathscr{A}}((q, u) \rightarrow *(p, w))=\mu_{\mathscr{A}}((q, v) \rightarrow *(p, w))$. This implies that for all $q, p \in Q \mu_{\mathscr{A}}((q, u) \rightarrow *(p, w))>$ $0_{L} \Longleftrightarrow \mu_{\mathscr{A}}((q, v) \rightarrow *(p, w))>0_{L}$, or $u \approx v$. Hence $[[u]]=[[v]]$ or $h([u])=h([v])$. This shows that $h$ is well-defined. Obviously $h$ is onto. Now for all $[u],[v] \in S(\mathscr{A})$, we have $f([u] \odot[v])=f([u \oplus v])=$ $[[u \oplus v]]=[[u]] \odot[[v]]=h([u]) \odot h([v])$. Since $S(\mathscr{A})$ is finite, therefore $\tilde{S}(\mathscr{A})$ is also finite.

Let $\mathscr{A}=(Q, \Sigma, \delta)$ be a fuzzy multiset finite automaton and $u \in \Sigma^{\oplus}$. For some $u, w \in \Sigma^{\oplus}$, define a fuzzy relation $u_{R}$ in $Q$ by $u_{R}(q, p)=\mu_{\mathscr{A}}((q, u) \rightarrow *(p, w))$, for all $q, p \in Q$. Further, let $Q, S, T$ be nonempty sets and $R, R^{\prime}$ be a fuzzy relation in $Q \times S$ and $S \times T$, respectively. Then the composite relation $R \circ R^{\prime}$ in $Q \times T$ is defined by;

$$
R \circ R^{\prime}(q, t)=\bigvee_{s \in S}\left\{R(q, s) \wedge R^{\prime}(s, t)\right\}, \forall q \in Q, t \in T .
$$

We use the composition of realtions $\circ$ defined above in the next proposition.

Proposition 3.6 Let $\mathscr{A}=(Q, \Sigma, \delta) \in \mathscr{F} \mathscr{M} \mathscr{A}\left(\Sigma^{\oplus}\right)$ and $S_{R}(\mathscr{A}):=\left\{u_{R} \mid u \in \Sigma^{\oplus}\right\}$. Then

(i) for all $u, v \in \Sigma^{\oplus}, u_{R} \circ v_{R}=[u \oplus v]_{R}$,

(ii) $\left\langle S_{R}(\mathscr{A}), \circ\right\rangle$ is a finite semigroup with identity and isomorphic to $\langle S(\mathscr{A}), \odot\rangle$.

Proof. $(i)$ Let $q, p \in Q$. Then

$[u \oplus v]_{R}(q, p)$

$$
\begin{aligned}
= & \mu_{\mathscr{A}}((q,[u \oplus v]) \rightarrow *(p, w)) \\
= & \bigvee_{r \in Q}\left\{\mu_{\mathscr{A}}((q, u) \rightarrow *(r, v)) \wedge\right. \\
& \left.\mu_{\mathscr{A}}((r, v) \rightarrow *(p, w))\right\} \\
= & \bigvee_{r \in Q}\left\{[u]_{R}(q, r) \wedge[v]_{R}(r, p)\right\} \\
= & \left([u]_{R} \circ[v]_{R}\right)(q, p) .
\end{aligned}
$$


Hence $u_{R} \circ v_{R}=[u \oplus v]_{R}$, for all $u, v \in \Sigma^{\oplus}$.

(ii) Clearly the binary operation $\circ$ is well-defined and associative with identity $0_{\Sigma_{R}}$. Since $Q$ and $\Sigma$ are finite, therefore $\left\langle S_{R}(\mathscr{A})\right\rangle$ is also finite. Now define a map $h: S_{R}(\mathscr{A}) \rightarrow S(\mathscr{A})$ by $h\left([u]_{R}\right)=[u]$, for all $[u]_{R} \in S_{R}(\mathscr{A})$. Let $[u]_{R},[v]_{R} \in S_{R}(\mathscr{A})$. Then

$$
\begin{aligned}
{[u]_{R}=[v]_{R} } & \\
& \Longleftrightarrow \quad[u]_{R}(q, p)=[v]_{R}(q, p), \forall q, p \in Q \\
& \Longleftrightarrow \mu_{\mathscr{A}}((q, u) \rightarrow *(p, w)) \\
& =\mu_{\mathscr{A}}((q, v) \rightarrow *(p, w)), \\
& \Longleftrightarrow[u]=[v] .
\end{aligned}
$$

Thus $h$ is well-defined and one-one. Obviously $h$ is onto. Now $h\left([u]_{R} \circ[v]_{R}\right)=h\left([u \oplus v]_{R}\right)=[u \oplus v]=[u] \odot$ $[v]=h\left([u]_{R}\right) \odot h\left([v]_{R}\right)$. Hence $h$ is a homomorphism. Therefore $\left\langle S_{R}(\mathscr{A}), \circ\right\rangle$ is isomorphic to $\langle S(\mathscr{A}), \odot\rangle$.

Definition 3.1 Let $\mathscr{A}=(Q, \Sigma, \delta) \in \mathscr{F} \mathscr{M} \mathscr{A}\left(\Sigma^{\oplus}\right)$ and $\simeq$ be an equivalence relation on $Q$. Then $\simeq$ is called an admissible relation if it satisfies: for all $q, p, r$ and $\forall a \in \Sigma, q \backsim p, \mu_{\mathscr{A}}\left((q,\langle a\rangle) \rightarrow *\left(r, 0_{\Sigma}\right)\right)>0_{L} \Rightarrow$ $\exists s \in Q$ such that $s \simeq r$ and $\mu_{\mathscr{A}}\left((p,\langle a\rangle) \rightarrow *\left(s, 0_{\Sigma}\right)\right) \geq$ $\mu_{\mathscr{A}}\left((q,\langle a\rangle) \rightarrow *\left(r, 0_{\Sigma}\right)\right)$.

Below we give the characterization of admissible relation in fuzzy multiset finite automata.

Proposition 3.7 Let $\mathscr{A}=(Q, \Sigma, \delta) \in \mathscr{F} \mathscr{M} \mathscr{A}\left(\Sigma^{\oplus}\right)$ and $\backsim$ be an equivalence relation on $Q$. Then $\backsim$ is admissible relation if and only if for all $q, p, r \in Q$ and $\forall v \subseteq u \in \Sigma^{\oplus}, q \backsim p, \mu_{\mathscr{A}}((q, u) \rightarrow *(r, v))>0_{L} \Rightarrow$ $\exists s \in Q$ such that $s \simeq r$ and $\mu_{\mathscr{A}}((p, u) \rightarrow *(s, v)) \geq$ $\mu_{\mathscr{A}}((q, u) \rightarrow *(r, v))$.

Proof. Let us assume that $\simeq$ is an admissible relation on $Q$. Let $q, p, r \in Q$ and $v \subseteq u \in \Sigma^{\oplus}$ such that $q \backsim p$ and $\mu_{\mathscr{A}}((q, u) \rightarrow *(r, v))>0_{L}$. We prove the result by method of induction on $|u|=n$. Now if $n=0$, then $u=0_{\Sigma}$ and so $\mu_{\mathscr{A}}((q, u) \rightarrow$ $\left.*\left(r, 0_{\Sigma}\right)\right)=\mu_{\mathscr{A}}\left(\left(q, 0_{\Sigma}\right) \rightarrow *\left(r, 0_{\Sigma}\right)\right)>0_{L} \Rightarrow q=r$ and $\mu_{\mathscr{A}}\left((q, u) \rightarrow *\left(q, 0_{\Sigma}\right)\right)=1_{L}$. If we take $s=p$, then $s \backsim r$ and $\mu_{\mathscr{A}}\left((p, u) \rightarrow *\left(s, 0_{\Sigma}\right)\right)=1_{L}=$ $\mu_{\mathscr{A}}\left((q, u) \rightarrow *\left(q, 0_{\Sigma}\right)\right)$. Hence the result is true for $n=0$. Suppose it is true for all $w \in \Sigma^{\oplus}$ with $|w|=n-1, n>0$. Let $u=w \oplus\langle a\rangle$, where $a \in \Sigma$. Then $\mu_{\mathscr{A}}\left((q, w \oplus\langle a\rangle) \rightarrow *\left(r, 0_{\Sigma}\right)\right)=\bigvee_{q_{1} \in Q}\left\{\mu_{\mathscr{A}}((q, w) \rightarrow\right.$ $\left.\left.*\left(q_{1},\langle a\rangle\right)\right) \wedge \mu_{\mathscr{A}}\left(\left(q_{1},\langle a\rangle\right) \rightarrow *\left(r, 0_{\Sigma}\right)\right)\right\}>0_{L}$. Let $s \in Q$ be such that $\mu_{\mathscr{A}}((q, w) \rightarrow *(s,\langle a\rangle)) \wedge$ $\mu_{\mathscr{A}}\left((s,\langle a\rangle) \rightarrow *\left(r, 0_{\Sigma}\right)\right)=\bigvee_{q_{1} \in Q}\left\{\mu_{\mathscr{A}}((q, w) \rightarrow\right.$ $\left.\left.*\left(q_{1},\langle a\rangle\right)\right) \wedge \mu_{\mathscr{A}}\left(\left(q_{1},\langle a\rangle\right) \rightarrow *\left(r, 0_{\Sigma}\right)\right)\right\}$. Then $\mu_{\mathscr{A}}((q, w) \rightarrow *(s,\langle a\rangle))>0_{L}, \quad \mu_{\mathscr{A}}((s,\langle a\rangle) \rightarrow$ $\left.*\left(r, 0_{\Sigma}\right)\right)>0_{L}$. It follows from the induction hypothesis that there exists $t_{s} \in Q$ such that $t_{s} \simeq$ $s, \mu_{\mathscr{A}}\left((p, w) \rightarrow *\left(t_{s},\langle a\rangle\right)\right) \geq \mu_{\mathscr{A}}((q, w) \rightarrow *(s,\langle a\rangle))$.
Now $\mu_{\mathscr{A}}\left((s,\langle a\rangle) \rightarrow *\left(r, 0_{\Sigma}\right)\right)>0$ and $t_{s} \simeq s$, then there exists $t \in Q$ such that $\mu_{\mathscr{A}}\left(\left(t_{s},\langle a\rangle\right) \rightarrow\right.$ $\left.*\left(t, 0_{\Sigma}\right)\right) \geq \mu_{\mathscr{A}}\left((s,\langle a\rangle) \rightarrow *\left(r, 0_{\Sigma}\right)\right)$ and $t \simeq p$. Thus $\mu_{\mathscr{A}}\left((p, w) \rightarrow *\left(t_{s},\langle a\rangle\right)\right) \wedge \mu_{\mathscr{A}}\left(\left(t_{s},\langle a\rangle\right) \rightarrow *\left(t, 0_{\Sigma}\right)\right) \geq$ $\mu_{\mathscr{A}}((q, w) \rightarrow *(s,\langle a\rangle)) \wedge \mu_{\mathscr{A}}\left((s,\langle a\rangle) \rightarrow *\left(r, 0_{\Sigma}\right)\right)$, for some $t \in Q$, and so $\mu_{\mathscr{A}}\left((q, w \oplus\langle a\rangle) \rightarrow *\left(r, 0_{\Sigma}\right)\right)=$ $\mu_{\mathscr{A}}((q, w) \rightarrow *(s,\langle a\rangle)) \wedge \mu_{\mathscr{A}}\left((s,\langle a\rangle) \rightarrow *\left(r, 0_{\Sigma}\right)\right) \leq$ $\mu_{\mathscr{A}}\left((p, w) \rightarrow *\left(t_{s},\langle a\rangle\right)\right) \wedge \mu_{\mathscr{A}}\left(\left(t_{s},\langle a\rangle\right) \rightarrow *\left(t, 0_{\Sigma}\right)\right) \leq$ $\bigvee_{r_{1} \in Q}\left\{\mu_{\mathscr{A}}\left((p, w) \rightarrow *\left(r_{1},\langle a\rangle\right)\right) \wedge \mu_{\mathscr{A}}\left(\left(r_{1},\langle a\rangle\right) \rightarrow\right.\right.$ $\left.\left.*\left(t, 0_{\Sigma}\right)\right)\right\}=\mu_{\mathscr{A}}\left((p, w \oplus\langle a\rangle) \rightarrow *\left(t, 0_{\Sigma}\right)\right)=$ $\mu_{\mathscr{A}}\left((p, u) \rightarrow *\left(t, 0_{\Sigma}\right)\right)$, and $t \bumpeq r$. Therefore the result is true for every $n, n=|u|, u \in \Sigma^{\oplus}$ by induction hypothesis. The converse of this is trivial and leave it to the reader.

Let $\mathscr{A}=(Q, \Sigma, \delta) \in \mathscr{F} \mathscr{M} \mathscr{A}\left(\Sigma^{\oplus}\right)$ and $\simeq$ be an admissible relation on $Q$. For $q \in Q$, let $[q]$ denote the equivalence class of $q$. Define a set $\tilde{Q}=\{[q] \mid q \in Q\}$. Let $\tilde{\delta}: \tilde{Q} \times \Sigma^{\oplus} \times \tilde{Q} \rightarrow L$ be a fuzzy state transition map whose configuration is defined as $\tilde{\mu}_{\mathscr{A}}(([q], u) \rightarrow$ $*([p], v))=\bigvee_{r \in[p]}\left\{\mu_{\mathscr{A}}((q, u) \rightarrow *(r, v))\right\}, \forall[q],[p] \in \tilde{Q}$ and $v \subseteq u \in \Sigma^{\oplus}$. Now we show that $\tilde{\delta}$ is a single-valued map. Let us suppose that $[q]=\left[q^{\prime}\right],[p]=\left[p^{\prime}\right], u=u^{\prime}$, $v=v^{\prime}, \forall q, q^{\prime}, p, p^{\prime} \in Q$ and $\forall u, u^{\prime}, v, v^{\prime} \in \Sigma^{\oplus}$. Then $q \simeq q^{\prime}$ and $p \simeq p^{\prime}$. Now

$\tilde{\mu}_{\mathscr{A}}(([q], u) \rightarrow *([p], v))$

$$
=\bigvee_{r \in[p]}\left\{\mu_{\mathscr{A}}((q, u) \rightarrow *(r, v))\right\}
$$

and

$\tilde{\mu}_{\mathscr{A}}\left(\left(\left[q^{\prime}\right], u^{\prime}\right) \rightarrow *\left(\left[p^{\prime}\right], v^{\prime}\right)\right)$

$$
=\bigvee_{r^{\prime} \in\left[p^{\prime}\right]}\left\{\mu_{\mathscr{A}}\left(\left(q^{\prime}, u\right) \rightarrow *\left(r^{\prime}, v\right)\right)\right\}
$$

Let $r \in[p]$ be such that $\mu_{\mathscr{A}}((q, u) \rightarrow *(r, v))>0_{L}$. Since $\simeq$ is admissible there exists $r^{\prime} \in Q$ such that $r^{\prime} \simeq r$ and $\mu_{\mathscr{A}}\left(\left(q^{\prime}, u\right) \rightarrow *\left(r^{\prime}, v\right)\right) \geq$ $\mu_{\mathscr{A}}((q, u) \rightarrow *(r, v))$. Now since $r^{\prime} \simeq r$, $r^{\prime} \in[p]=\left[p^{\prime}\right]$. Thus there exists $r^{\prime} \in\left[p^{\prime}\right]$ such that $\mu_{\mathscr{A}}\left(\left(q^{\prime}, u\right) \rightarrow *\left(r^{\prime}, v\right)\right) \geq \mu_{\mathscr{A}}((q, u) \rightarrow *(r, v))>0_{L}$. Similarly if $\mu_{\mathscr{A}}\left(\left(q^{\prime}, u\right) \rightarrow *\left(r^{\prime}, v\right)\right)>0_{L}$ for some $r^{\prime} \in\left[p^{\prime}\right]$, then there exists $r \in[p]$ such that $\mu_{\mathscr{A}}((q, u) \rightarrow *(r, v)) \geq \mu_{\mathscr{A}}\left(\left(q^{\prime}, u\right) \rightarrow *\left(r^{\prime}, v\right)\right)>0_{L}$. Hence $\mu_{\mathscr{A}}\left(\left(q^{\prime}, u\right) \rightarrow *\left(r^{\prime}, v\right)\right)=\mu_{\mathscr{A}}((q, u) \rightarrow *(r, v))$ or $\tilde{\mu}_{\mathscr{A}}(([q], u) \rightarrow *([p], v))=\tilde{\mu}_{\mathscr{A}}\left(\left(\left[q^{\prime}\right], u^{\prime}\right) \rightarrow *\left(\left[p^{\prime}\right], v^{\prime}\right)\right)$. Thus $\tilde{\delta}$ is a single-valued map. Hence $\tilde{\mathscr{A}}=(\tilde{Q}, \Sigma, \tilde{\delta})$ is a fuzzy multiset finite automaton. Now we define a $\operatorname{map} \tilde{h}: Q \rightarrow \tilde{Q}$ by $\tilde{h}(q)=[q], \forall q \in Q$. Clearly $\tilde{h}$ maps $Q$ onto $\tilde{Q}$. Let $q, p \in Q$ and $v \subseteq u \in \Sigma^{\oplus}$. Then 


$$
\begin{aligned}
\tilde{\mu}_{\mathscr{A}^{\prime}}(([q], u) & \rightarrow *([p], v)) \\
& =\bigvee_{r \in[p]}\left\{\mu_{\mathscr{A}}((q, u) \rightarrow *(r, v))\right\} ; \\
& \geq \mu_{\mathscr{A}}((q, u) \rightarrow *(p, v)),
\end{aligned}
$$

i.e., $\quad \mu_{\mathscr{A}}((q, u) \rightarrow *(p, v)) \leq \tilde{\mu}_{\mathscr{A}^{\prime}}((\tilde{h}(q), u) \rightarrow$ $*(\tilde{h}(p), v))$. Hence $\tilde{h}$ is a strong homomorphism.

Definition 3.2 Let $\mathscr{A}=(Q, \Sigma, \delta)$ and $\mathscr{A}^{\prime}=$ $\left(Q^{\prime}, \Sigma, \delta^{\prime}\right) \in \mathscr{F} \mathscr{M} \mathscr{A}\left(\Sigma^{\oplus}\right)$ and let $h: \mathscr{A} \rightarrow \mathscr{A}^{\prime}$ be strong homomorphism. Then the kernel of $h\left(\mathbf{K e r}_{\mathbf{h}}\right)$ defined by $\mathbf{K e r}_{\mathbf{h}}=\{(q, p) \mid h(q)=h(p)\}$.

Now we have the following.

Proposition 3.8 Let $\quad \mathscr{A}=(Q, \Sigma, \delta) \quad$ and $\mathscr{A}^{\prime}=\left(Q^{\prime}, \Sigma, \delta^{\prime}\right) \in \mathscr{F} \mathscr{M} \mathscr{A}\left(\Sigma^{\oplus}\right)$ and $h: \mathscr{A} \rightarrow \mathscr{A}^{\prime}$ be strong homomorphism. Then $\mathbf{K e r}_{\mathbf{h}}$ is an admissible relation.

Proof. Clearly $\mathrm{Ker}_{\mathbf{h}}$ is an equivalence relation on $Q$. Let $q, p \in Q$ be such that $(q, p) \in \mathbf{K e r}_{\mathbf{h}}$. Then $h(q)=h(p)$. Let $r \in Q, v \subseteq u \in \Sigma^{\oplus}$ and $\mu_{\mathscr{A}}((q, u) \rightarrow$ $*(r, v))>0_{L} . \quad$ Then $\mu_{\mathscr{A ^ { \prime }}}^{\prime}((h(q), u) \rightarrow *(h(r), v))=$ $\mu_{\mathscr{A}^{\prime}}^{\prime}((h(p), u) \rightarrow *(h(r), v)) \geq \mu_{\mathscr{A}}((p, u) \rightarrow *(r, v))$. Now by Proposition 2.2 there exists $r^{\prime} \in Q$ such that $\mu_{\mathscr{A}}\left((q, u) \rightarrow *\left(r^{\prime}, v\right)\right) \geq \mu_{\mathscr{A}}((p, u) \rightarrow *(r, v))>0_{L}$ and $h\left(r^{\prime}\right)=h(r)$. Since $h\left(r^{\prime}\right)=h(r),\left(r^{\prime}, r\right) \in \mathbf{K e r}_{\mathbf{h}}$. Thus $\mathbf{K e r}_{\mathbf{h}}$ is an admissible relation.

Proposition 3.9 Let $\mathscr{A}=(Q, \Sigma, \delta)$ and $\mathscr{A}^{\prime}=$ $\left(Q^{\prime}, \Sigma, \delta^{\prime}\right) \in \mathscr{F} \mathscr{M} \mathscr{A}\left(\Sigma^{\oplus}\right)$ and let $h: \mathscr{A} \rightarrow \mathscr{A}^{\prime}$ be strong homomorphism. Then there exists an isomorphism $g: \tilde{A}\left(=\left(Q / \mathbf{K e r}_{\mathbf{h}}, \Sigma, \tilde{\boldsymbol{\delta}}\right)\right) \rightarrow \mathscr{A}^{\prime}\left(=\left(Q^{\prime}, \Sigma, \boldsymbol{\delta}^{\prime}\right)\right)$ such that $h=g \circ \tilde{h}$.

Proof. We define map $g: Q / \mathbf{K e r}_{\mathbf{h}} \rightarrow Q^{\prime}$ by $g([q])=h(q)$. Let $q, p \in Q$ be such that $[q]=[p]$. Then $(q, p) \in$ Ker $_{\mathbf{h}}$ and hence $g([q])=h(q)=h(p)=g([p])$. Thus $g$ is well-defined. Also, we can easily check that $g$ is one-one and onto. Now, let $v \subseteq u \in \Sigma^{\oplus}$. Then

$$
\begin{aligned}
\mu_{\mathscr{A}} & (([q], u) \rightarrow *([p], v)) \\
& =\bigvee\left\{\mu_{\mathscr{A}}((q, u) \rightarrow *(r, v)) \mid r \in[p]\right\} ; \\
& =\bigvee\left\{\mu_{\mathscr{A}}((q, u) \rightarrow *(r, v)) \mid h(r)=h(p), r \in Q\right\} ; \\
& =\mu_{\mathscr{A}^{\prime}}((h(q), u) \rightarrow *(h(p), v)) ; \\
& =\mu_{\mathscr{A}^{\prime}}((g(q), u) \rightarrow *(g(p), v)) .
\end{aligned}
$$

Thus $g$ is homomorphism and hence isomorphism from $Q / \mathbf{K e r}_{\mathbf{h}}$ to $Q^{\prime}$. Obviously, $h=g \circ \tilde{h}$.

\section{Conclusion}

In this paper, we have tried to characterize quotient structures of fuzzy multiset finite automata. We introduced different congruence relation on multiset associated with fuzzy multiset finite automata and show that each congruence relation associates a semigroup with the fuzzy multiset finite automata. Moreover, we have introduced an admissible relation on a fuzzy multiset finite automata and discuss its characterization. Finally, we established an isomorphism between fuzzy multiset finite automata and the quotient structures on another fuzzy multiset finite automata.

As a future work we plan to define and study the operator oriented fuzzy multiset finite automata and its association with fuzzy multiset topologies.

\section{Acknowledgement}

The work of first and third author is supported through the ERDF/ESF project AI-Met4AI No. CZ.02.1.01/0.0/0.0/17_049/0008414.

\section{References}

[1] F. Blanchet-Sadri, C. Morgan, Multiset and set decipherable codes, Computers \& Mathematics with Applications 41 (10-11) (2001) 1257-1262.

[2] W. D. Blizard, et al., Multiset theory., Notre Dame Journal of formal logic 30 (1) (1988) 3666.

[3] M. Cavaliere, R. Freund, M. Oswald, D. Sburlan, Multiset random context grammars, checkers, and transducers, Theoretical computer science 372 (2-3) (2007) 136-151.

[4] G. Ciobanu, M. Gontineac, Mealy multiset automata, International Journal of Foundations of Computer Science 17 (01) (2006) 111-126.

[5] G. Ciobanu, V. M. Gontineac, Algebraic and coalgebraic aspects of membrane computing, in: International Workshop on Membrane Computing, Springer, 2005, pp. 181-198.

[6] E. Csuhaj-Varjú, C. Martin-Vide, V. Mitrana, Multiset automata, in: Workshop on Membrane Computing, Springer, 2000, pp. 69-83.

[7] J. Jin, Q. Li, Y. Li, Algebraic properties of Lfuzzy finite automata, Information Sciences 234 (2013) 182-202.

[8] Y. B. Jun, Quotient structures of intuitionistic fuzzy finite state machines, Information Sciences 177 (22) (2007) 4977-4986.

[9] J. Močkoř, Fuzzy and non-deterministic automata, Soft Computing 3 (4) (1999) 221-226. 
[10] J. N. Mordeson, D. S. Malik, Fuzzy automata and languages: theory and applications, CRC Press, 2002.

[11] V. K. Murthy, E. Krishnamurthy, Probabilistic parallel programming based on multiset transformation, Future generation Computer systems 11 (3) (1995) 283-293.

[12] P. Pal, S. P. Tiwari, Category of L-valued multiset automata and Brzozowski's algorithm, New Mathematics and Natural Computation 16 (03) (2020) 481-496.

[13] D. Qiu, Characterizations of fuzzy finite automata, Fuzzy Sets and Systems 141 (3) (2004) 391-414.

[14] E. S. Santos, Maximin automata, Information and Control 13 (4) (1968) 363-377.

[15] M. Shamsizadeh, M. Zahedi, A note on quotient structures of intuitionistic fuzzy finite state machines, Journal of Applied Mathematics and Computing 51 (1-2) (2016) 413-423.

[16] B. Sharma, A. Syropoulos, S. P. Tiwari, On fuzzy multiset regular grammars, Annals of Fuzzy Mathematics and Informatics 12 (5) (2016) 617639.

[17] B. K. Sharma, V. Gautam, S. P. Tiwari, V. Bhattacherjee, On fuzzy multiset regular languages, Journal of Fuzzy Set Valued Analysis 2017 (1) (2017) 50-61.

[18] B. K. Sharma, S. P. Tiwari, S. Sharan, On algebraic study of fuzzy multiset finite automata, Fuzzy Information and Engineering 8 (3) (2016) 315-327.

[19] S. P. Tiwari, V. Gautam, M. Dubey, On fuzzy multiset automata, Journal of Applied Mathematics and Computing 51 (1-2) (2016) 643-657.

[20] P. Martinek, A simplified form of fuzzy multiset finite automata, Artificial Intelligence Perspectives in Intelligent Systems 464 (2016) 469-476.

[21] J. Wang, M. Yin, W. Gu, Fuzzy multiset finite automata and their languages, Soft Computing 17 (3) (2013) 381-390.

[22] Y. Wang, Y. Li, Minimization of lattice multiset finite automata, Journal of Intelligent \& Fuzzy Systems 35 (1) (2018) 627-637.

[23] W. G. Wee, On generalizations of adaptive algorithms and application of the fuzzy sets concept to pattern classification.
[24] W. G. Wee, K.-S. Fu, A formulation of fuzzy automata and its application as a model of learning systems, IEEE Transactions on Systems Science and cybernetics 5 (3) (1969) 215-223.

[25] L. A. Zadeh, Fuzzy sets, Information and control 8 (3) (1965) 338-353. 